\title{
Asteroseismology in the BINA context
}

\author{
Anwesh Mazumdar ${ }^{1}$, Bhaswati Mookerjea ${ }^{2}$, Pritesh Ranadive ${ }^{1}$
}

\author{
${ }^{1}$ Homi Bhabha Centre for Science Education (TIFR) \\ V. N. Purav Marg, Mumbai 400088, India. \\ ${ }^{2}$ Dept. of Astronomy and Astrophysics, Tata Institute of Fundamental Research \\ Homi Bhabha Road, Mumbai 400005, India.
}

\begin{abstract}
The scope of asteroseismic studies within the Belgo-Indian Network for Astronomy and astrophysics (BINA) is explored. The main strands of current research in asteroseismology of solar-type stars are outlined. While it is obvious that it would be difficult to match the quality of the space-based seismic data on such stars with ground-based telescopes, the possibility of contribution to supplemental non-seismic data is considered. New calibrations of colour-temperature relations using archival data are presented. It is proposed that with similar calibrations with BINA-based photometric data in the future, it would be possible to provide accurate estimates of effective temperatures of solar-type stars that would be useful in asteroseismology of such stars.
\end{abstract}

Keywords: asteroseismology - solar-type stars - photometry - colour - effective temperature

\section{Introduction}

Asteroseismology or the study of oscillations in stars is, by far, the most powerful test of the theory of stellar interiors and stellar evolution. It is the only probe that can "pierce through the outer layers of a star and test the conditions within" (Eddington 1926). In recent years, this field has received an enormous boost due to the excellent data on minute oscillations collected by several space missions, namely, CoRoT, Kepler and TESS.

Oscillations in stars appear mainly as two kinds of standing waves, characterized as p-modes and g-modes. The so-called p-modes are primarily acoustic in nature with gradient of pressure acting as the restoring force. The other type, g-modes, are internal gravity waves, where buoyancy provides the restoring force. Under certain situations, modes of mixed character may appear, which behave as g-modes near the core of the star and as p-modes in the exterior layers. The oscillations in stars can also be classified in two broad types, depending on the physics of the excitation mechanism. The comparatively larger amplitude oscillations, seen mostly in stars of higher mass, are the so-called opacity-driven oscillations. Solar-type stars, i.e., low mass main sequence stars, exhibit multimodal low amplitude oscillations which are excited by the turbulence in their outer convection zones. These are termed solar-like oscillations. Red giant stars, which also possess large convective outer layers, exhibit such stochastically driven oscillations as well. In this work we concentrate on solar-type stars which exhibit rich multi-mode oscillation spectra of low angular degree. Modes of higher degree cannot be observed in distant stars due to a geometric cancellation effect. However, it turns out that the low degree modes are most suited for probing the conditions of the central regions of stars. 
Here we explore how the telescopes under the BINA consortium can be utilized for asteroseismic studies of solar-type stars. While we recognize that it would be difficult to match the quality and quantity of the seismic data now available from several space missions, we consider the possibility of BINA telescopes being fruitfully used in providing accurate non-seismic constraints for solar type targets observed by the space telescopes.

\section{Asteroseismology of solar-type stars}

The principal strands of current research in asteroseismology of solar-type stars appear to be

1. determination of mass and radius from scaling relations,

2. detailed forward modelling,

3. analysis of acoustic glitches.

There are, of course, other areas of interest in the asteroseismology of solar-type stars, principal among them being the study of rotation of the stellar interior from splittings in the frequencies. Another avenue of research is through inversion methods. However, although inversion has been spectacularly successful in helioseismology, not a great deal of success has been achieved so far for distant stars. Parallelly, a great deal of progress has been made in recent years in the data reduction and analysis techniques of seismic observations. Here we provide a very brief outline of the above three strands of research in asteroseismology of solar-type stars. For a comprehensive review, see Chaplin \& Miglio (2013).

\subsection{Mass and radius from scaling relations}

The asymptotic theory of stellar oscillations, which is valid for high radial order modes, connects the mean density, surface gravity and the effective temperature of a star to seismic observables such as the mean large separation or radial modes, $\Delta_{0}$, and the frequency of maximum power, $\nu_{\max }$. Using this theory, the mass and radius of a star can be determined directly from its frequency spectrum (see e.g., Chaplin \& Miglio 2013):

$$
\begin{aligned}
\frac{M}{M_{\odot}} & \approx\left(\frac{\nu_{\max }}{\nu_{\max , \odot}}\right)^{3}\left(\frac{\Delta_{0}}{\Delta_{0, \odot}}\right)^{-4}\left(\frac{T_{\mathrm{eff}}}{T_{\mathrm{eff}, \odot}}\right)^{3 / 2} \\
\frac{R}{R_{\odot}} & \approx\left(\frac{\nu_{\max }}{\nu_{\text {max }, \odot}}\right)\left(\frac{\Delta_{0}}{\Delta_{0, \odot}}\right)^{-2}\left(\frac{T_{\mathrm{eff}}}{T_{\mathrm{eff}, \odot}}\right)^{1 / 2}
\end{aligned}
$$

These relations are the basis of the so-called grid modelling technique where a large grid of stellar models is used to search for a close match of these seismic observables along with other spectroscopic constraints, in particular, $T_{\text {eff }}$ and [Fe/H] (Gai et al. 2011). Further, if a reliable estimate of luminosity through parallax measurement is available, then that is also used as an additional constraint. Overall, grid modelling has, so far, provided quite robust estimates of mass and radius of stars, which have been particularly useful in the case of planet-hosting stars to characterize the detected planets. 


\subsection{Detailed forward modelling}

While grid modelling can often lead to reliable determination of mass and radius of a star, it cannot very well provide a stringent test of the physics of the stellar interior. The $\Delta_{0}$ or $\nu_{\max }$ only characterize the overall nature of the stellar oscillations. But the oscillation spectrum actually encodes much more information in the form of individual frequencies. These frequencies can be compared with the frequencies of theoretical models to determine the structure and composition of the stellar interior, and thereby the global properties as well. Of course, such forward modelling also requires non-seismic constraints such as $T_{\text {eff }}$ and $[\mathrm{Fe} / \mathrm{H}]$ to be specified.

\subsection{Acoustic glitch analysis}

Both the grid modelling and detailed forward modelling techniques are dependent on the details of the input physics of the stellar models, the latter especially so. However, even if a complete profile of the stellar interior may not be obtained without detailed modelling, one can extract information about particular layers inside a star from certain specific features of the frequency spectrum. These relate to so-called acoustic glitches - layers inside a star where the sound speed undergoes an abrupt change due to sharp changes in the structure. Each glitch produces an oscillatory signature in the frequencies, whose periodicity is related to the acoustic depth of the glitch (Vorontsov 1988, Gough 1990). Indeed, an analysis of this oscillatory signal yields model-independent information about the specific layers with sharp features (Mazumdar et al. 2012).

The principal acoustic glitches inside a star are the boundaries of convective regions and partial ionization zones. Although the oscillatory signal from each glitch should, in principle, be present in the frequencies, the actual detection of such a signal depends both on the amplitude of the signal as well as the acoustic location of the glitch inside the star. A glitch that lies deep inside a star, e.g., at the boundary of the convective core in stars slightly more massive than the Sun, is hard to extract from the frequencies due to an aliasing effect (Mazumdar \& Antia 2001). On the other hand, even a strong signal from a superficial layer, from the hydrogen ionisation zone, cannot be isolated because the periodicity of the corresponding oscillatory signal exceeds the range of frequencies that can be observed in a typical star. In effect, usually two oscillatory signals can be extracted from the frequency spectrum: one is from the base of the outer convection zone, and the other is from the local maximum in the profile of the adiabatic exponent between the first and second helium ionisation zones (Mazumdar et al. 2014, Verma et al. 2014). The amplitude of the helium glitch signal can be calibrated with stellar models to estimate the helium abundance in stellar envelopes of solar-type stars. Indeed, such analyses have been carried out for a large number of Kepler stars (Verma et al. 2017, 2019).

\section{BINA and asteroseismology}

The asteroseismic studies of solar-type stars, as outlined above, can only be undertaken if the frequencies of oscillations can be reliably determined from long time series photometry. At present, oscillations with relative amplitudes as low as few ppm in luminous flux can be detected in the ultra-precise space-based photometric data, and the continuous observation windows in space afford an uncertainty in frequencies $\sim 0.1 \mu \mathrm{Hz}$. While this is likely to improve further in the future with advances in technology as well as data reduction techniques, the quality of even the present space-based seismic data cannot be matched by long observing campaigns undertaken at ground-based facilities. However, as discussed above, for a complete asteroseismic analysis, the seismic data must be supplemented by parameters such as $T_{\text {eff }}$ and $[\mathrm{Fe} / \mathrm{H}]$, which are determined from high resolution spectra obtained on 
the ground. But even then, the requirement of spectroscopic data for the very large number of target stars observed by the space missions places a large demand on the ground-based telescopes. In this context, faster methods to reliably estimate the $T_{\text {eff }}$ of stars would be useful. For example, the $T_{\text {eff }}$ can be estimated from a well-calibrated colour-temperature relation applied on multi-band photometric measurements. This is where BINA telescopes can be useful in the context of asteroseismology, especially of solar-type stars.

We propose that the BINA telescopes can be used to obtain precise multi-band photometric data of the solar-type target stars of space missions such as Kepler, TESS or PLATO, which can then be used to estimate their $T_{\text {eff }}$ through a well-calibrated colour-temperature relation. Of course, this would only be useful if the typical uncertainties in the $T_{\text {eff }}$ values so obtained are comparable to that from spectroscopic measurements. We explore the feasibility of such a method in the next section.

\section{Colour-temperature relations}

Since large scale multi-band photometric data is not yet available from the BINA telescopes, we use photometric data from other sources for testing calibration relations. The temperatures are taken from published catalogues of spectroscopically obtained $T_{\text {eff. }}$. We focus on $\mathrm{F}, \mathrm{G}$ and $\mathrm{K}$ type stars which constitute the solar-type population.

Among the many colour indices that may be constructed from multi-band photometry, it has been found that the colour $V-K$ is a particularly robust indicator of $T_{\text {eff }}$ (Casagrande et al. 2010). The rms residual of this index from a suitable calibration curve has been found to be the least among several indices. Two vast sources of $V$ and $K$ magnitudes of FG stars are the Tycho and 2MASS catalogues, respectively.

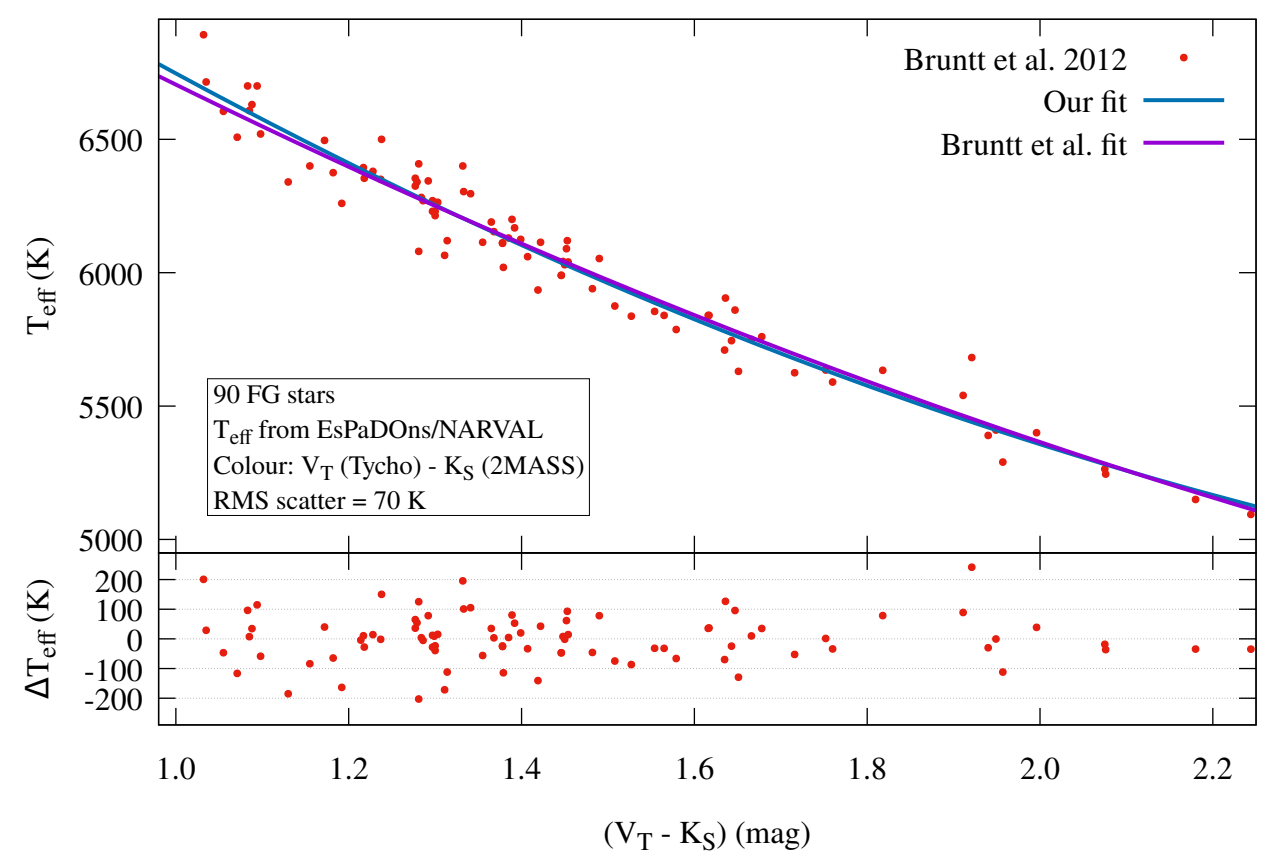

Figure 1: The calibration curve of $T_{\text {eff }}$ against the colour index $\left(V_{\mathrm{T}}-K_{\mathrm{S}}\right)$ for a sample of $90 \mathrm{~F}$ and $\mathrm{G}$ stars from Bruntt et al. (2012). The residuals are shown in the bottom panel. The relation obtained by Bruntt et al. (2012) is also shown.

As a calibration relation, we choose a quadratic form, inspired by Bruntt et al. (2012),

$$
T_{\text {eff }} / \mathrm{K}=a\left(V_{\mathrm{T}}-K_{\mathrm{S}}\right)^{2}+b\left(V_{\mathrm{T}}-K_{\mathrm{S}}\right)+c
$$


where $V_{\mathrm{T}}$ are the Tycho $V$ and $K_{\mathrm{S}}$ are the 2MASS $K$ magnitudes, respectively, and $a, b$ and $c$ are coefficients to be determined. In our first exercise, the $T_{\text {eff }}$ values are taken from Bruntt et al. (2012) who used the ESPaDOnS and NARVAL spectroscopic data (Donati et al. 2006). For a sample of 90 stars with $T_{\text {eff }}$ between $5000 \mathrm{~K}$ and $7000 \mathrm{~K},\left(V_{\mathrm{T}}-K_{\mathrm{S}}\right)$ between 1.0 and $2.2 \mathrm{mag}, \log g$ between 3.7 and 4.5 , and $[\mathrm{Fe} / \mathrm{H}]$ between $-0.6 \mathrm{dex}$ and $+0.4 \mathrm{dex}$, we find the following calibration relation

$$
T_{\text {eff }} / \mathrm{K}=365\left(V_{\mathrm{T}}-K_{\mathrm{S}}\right)^{2}-2485\left(V_{\mathrm{T}}-K_{\mathrm{S}}\right)+8867
$$

with a rms scatter of about $70 \mathrm{~K}$. The curve is shown in Fig. 1 along with the data and residuals. Our relation differs slightly from that found by Bruntt et al. (2012) for the same sample, which is also shown in the same figure. The fits were carried out under the assumption of uniform uncertainties for both $\left(V_{\mathrm{T}}-K_{\mathrm{S}}\right)$ and $T_{\text {eff }}$ values, and therefore, uncertainties in the data are not shown.

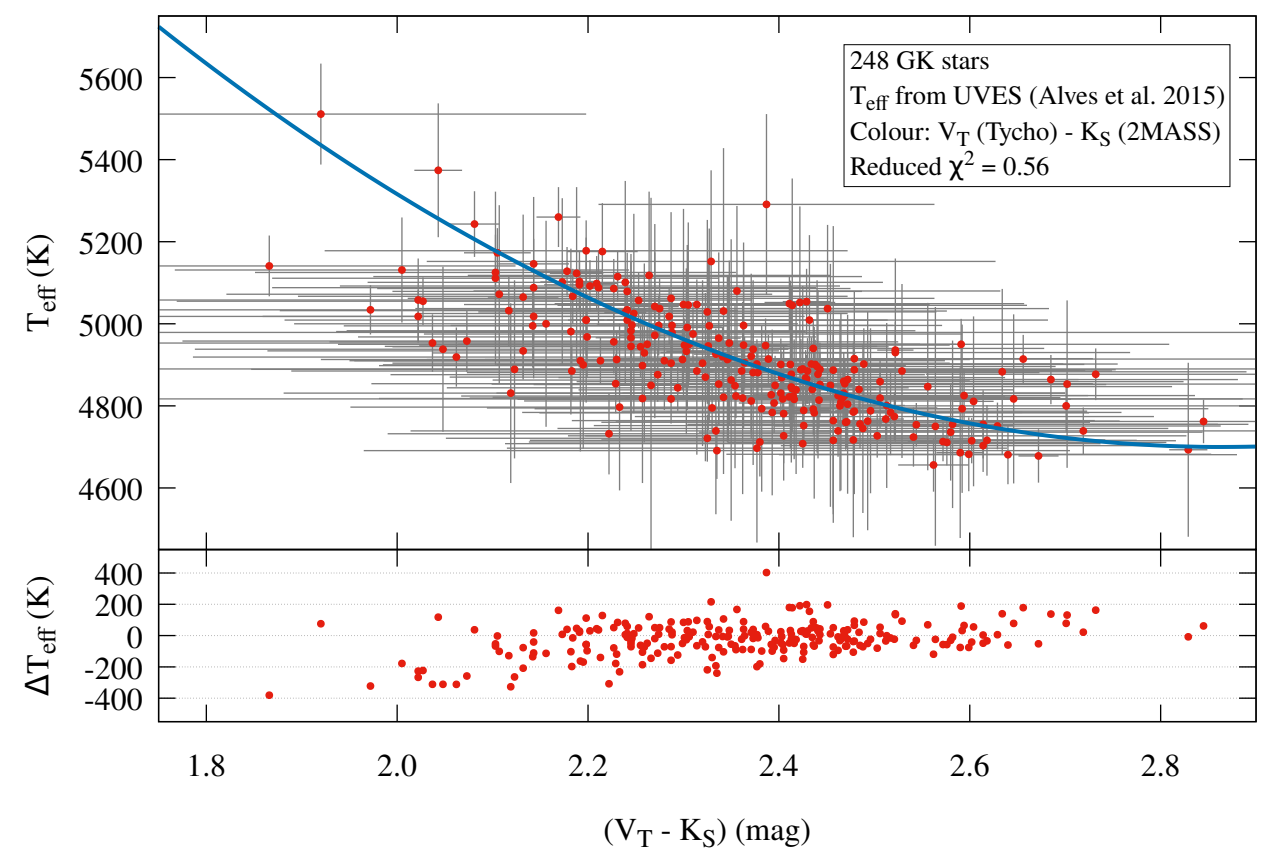

Figure 2: The calibration curve of $T_{\text {eff }}$ against the colour index $\left(V_{\mathrm{T}}-K_{\mathrm{S}}\right)$ for a sample of $248 \mathrm{G}$ and $\mathrm{K}$ stars with $T_{\text {eff }}$ from Alves et al. (2015). The residuals are shown in the bottom panel.

We also calibrated a similar colour-temperature relation at lower temperatures between $4000 \mathrm{~K}$ and $5500 \mathrm{~K}$ with a different sample of $248 \mathrm{G}$ and $\mathrm{K}$ stars which have $\left(V_{\mathrm{T}}-K_{\mathrm{S}}\right)$ between 1.0 and $3.5 \mathrm{mag}, \log g$ between 2.3 and 3.8 , and $[\mathrm{Fe} / \mathrm{H}]$ between $-0.7 \mathrm{dex}$ and $+0.4 \mathrm{dex}$. The $T_{\text {eff }}$ for these were determined from UVES spectra by Alves et al. (2015) and the colour index was again taken as $\left(V_{\mathrm{T}}-K_{\mathrm{S}}\right)$. The data and the following fit are shown in Fig. 2.

$$
T_{\text {eff }} / \mathrm{K}=825\left(V_{\mathrm{T}}-K_{\mathrm{S}}\right)^{2}-4726\left(V_{\mathrm{T}}-K_{\mathrm{S}}\right)+11468
$$

Finally, we obtained a colour-temperature relation for a sample of $451 \mathrm{~F}, \mathrm{G}$ and $\mathrm{K}$ stars. The $T_{\text {eff }}$ for these were taken from Sousa et al. (2016) who used HARPS spectra and reported an uncertainty of $76 \mathrm{~K}$ in the $T_{\text {eff }}$, on average. For this sample, spanning a range of $T_{\text {eff }}$ from $4500 \mathrm{~K}$ to $7000 \mathrm{~K}$, $\left(V_{\mathrm{T}}-K_{\mathrm{S}}\right)$ between 1.0 and $3.5 \mathrm{mag}, \log g$ between 3.7 and 5.0 , and $[\mathrm{Fe} / \mathrm{H}]$ between $-0.5 \mathrm{dex}$ and +1.0 dex, we find the following calibration (see Fig. 3):

$$
T_{\text {eff }} / \mathrm{K}=528\left(V_{\mathrm{T}}-K_{\mathrm{S}}\right)^{2}-3249\left(V_{\mathrm{T}}-K_{\mathrm{S}}\right)+9672
$$


The uncertainty in the coefficients of the quadratic relation in Eq. 6 is $\sim 2 \%$, which would translate into an uncertainty of about $100 \mathrm{~K}$ in derived $T_{\text {eff }}$ for a given $\left(V_{\mathrm{T}}-K_{\mathrm{S}}\right)$ index of a star. Therefore, in order to achieve a precision of about $70 \mathrm{~K}$ (which is the usual uncertainty in $T_{\text {eff }}$ values derived spectroscopically), one needs to improve slightly on the precision of the photometric measurements.

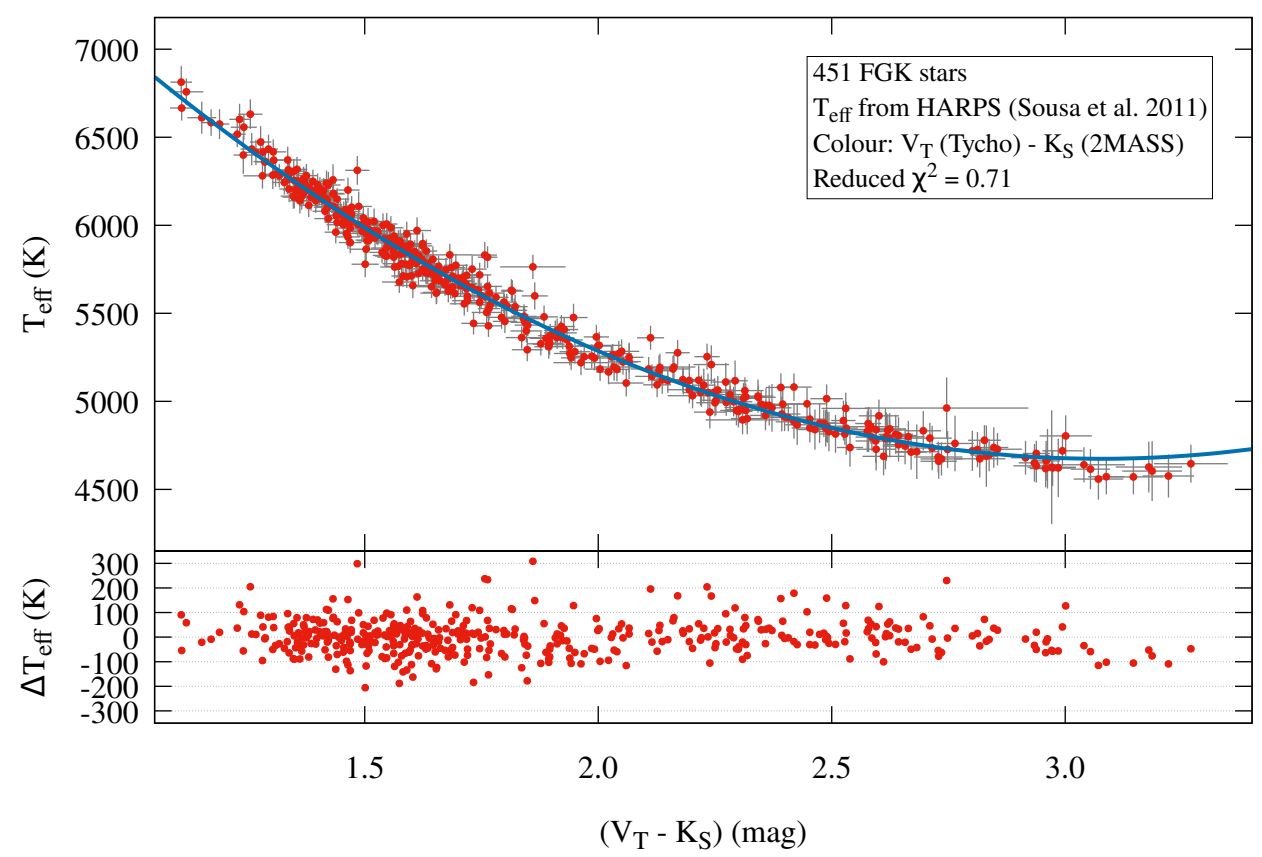

Figure 3: The calibration curve of $T_{\text {eff }}$ against the colour index $\left(V_{\mathrm{T}}-K_{\mathrm{S}}\right)$ for a sample of $451 \mathrm{~F}, \mathrm{G}$ and K stars with $T_{\text {eff }}$ from Sousa et al. (2016). The residuals are shown in the bottom panel.

It is interesting to check how the calibration relation of Eq. 6 fares for the other two samples used in Eqs. 4 and 5. We have made this comparison in Fig. 4. We note that the calibration works reasonably well with these samples as well, although there is a slight systematic shift for both samples. This is not surprising, given the different ranges of other spectroscopic parameters $([\mathrm{Fe} / \mathrm{H}]$ and $\log g)$ of these samples. The exact choice of the calibration relation thus depends slightly on the parameters of the stars of interest. Overall, Eq. 6 appears to work best for a large range of parameters. The slightly higher value of the reduced $\chi^{2}$ for Eq. 6 does not necessarily make it inferior to Eq. 5 because the lower value for the latter is primarily due to the considerably large estimates for uncertainty in both $T_{\text {eff }}$ and colour. Nevertheless, it is clear that a robust colour-temperature relation, as in Eq. 6, can be used to obtain reliable $T_{\text {eff }}$ values from multi-band photometry.

\section{Conclusions}

In this work, we have explored how the BINA collaboration can be used effectively to contribute to asteroseismology of solar-type stars. While it would be difficult for BINA telescopes to match the quality of the photometric data already available from space missions such as Kepler and TESS, they can be utilised in the ground-based support that is needed to supplement the seismic data. We propose that multi-band photometry from BINA telescopes be used to determine the $T_{\text {eff }}$ of $\mathrm{F}, \mathrm{G}$ and $\mathrm{K}$ stars via a suitably calibrated colour-temperature relation. While we do not yet have sufficient data from BINA to carry out this calibration, we have demonstrated through similar calibrations with archival photometric and temperature data that it is indeed possible to obtain robust estimates of $T_{\text {eff }}$ through this method. In order to attain a typical precision of around $70 \mathrm{~K}$ in $T_{\text {eff }}$, as usually available in current 


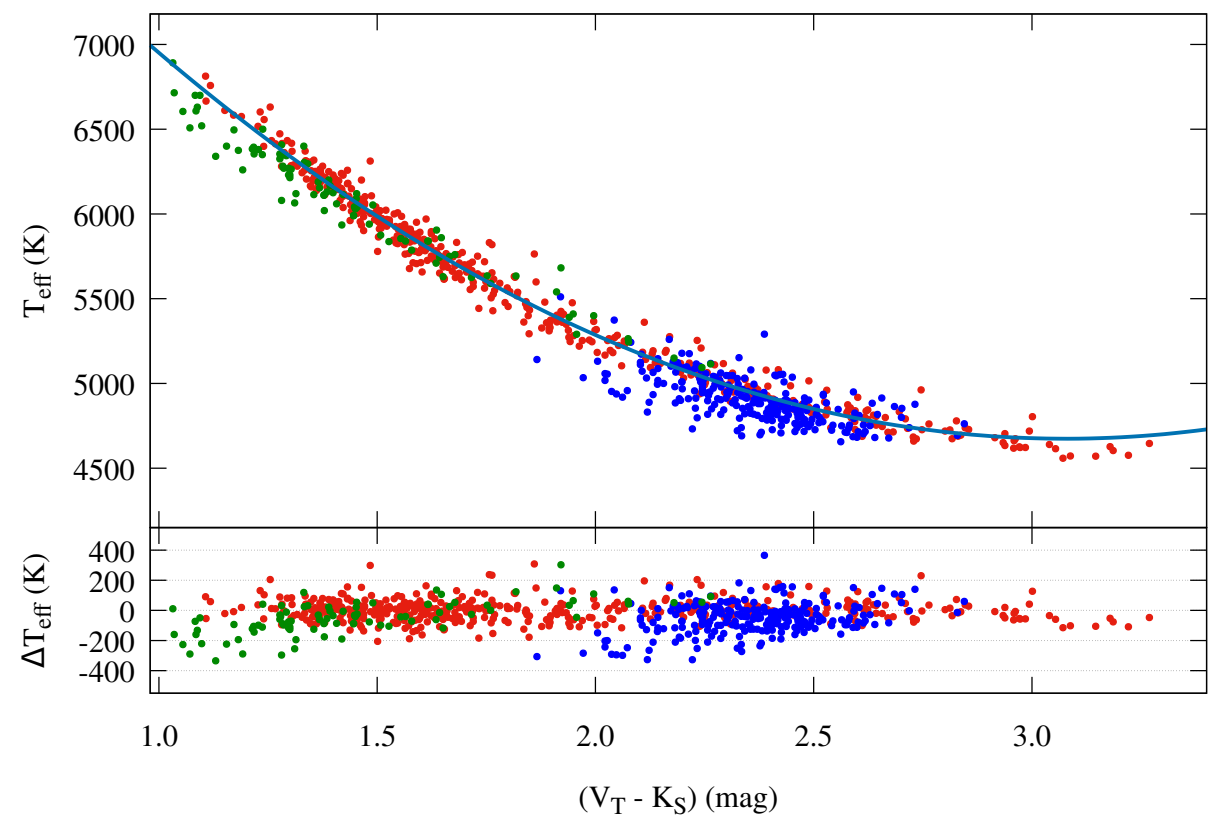

Figure 4: The calibration curve (Eq. 6) of $T_{\text {eff }}$ against the colour index $\left(V_{\mathrm{T}}-K_{\mathrm{S}}\right)$ is compared for the three samples from Fig. 1 (green dots), Fig. 2 (blue dots) and Fig. 3 (red dots). The residuals are shown in the bottom panel.

asteroseismic modelling efforts, we need a photometric precision of better than 0.02 mag. This should be possible with the full realization of the current instrumentation efforts at the BINA telescopes.

\section{Acknowledgements}

We thank the organisers of the 2nd BINA workshop for the opportunity to present this work, and DST for the grant DST/INT/Belg/P-02 that enabled travel for AM to the workshop. AM and PR would also like to thank the NIUS project under HBCSE for support.

\section{References}

Alves S., Benamati L., Santos N. C. et al. 2015, MNRAS, 448, 2749

Bruntt H., Basu S., Smalley B., Chaplin W. J. et al. 2012, MNRAS, 423, 122

Casagrande L., Ramírez I., Meléndez J., Bessell M., Asplund M. 2010, A\&A, 512, A54

Chaplin W. J., Miglio, A. 2013, ARA\&A, 51, 353

Donati J.-F., Catala C., Landstreet J. D. et al. 2006, ASP Conf. Ser., 358, 362

Eddington A. S. 1926, in Internal Constitution of Stars, Cambridge University Press (ISBN 9780521337083)

Gai N., Basu S., Chaplin W. J., Elsworth Y. 2011, ApJ, 730, 63

Gough D. O. 1990, LNP, 367, 283

Mazumdar A., Antia H. M. 2001, A\&A, 540, A31

Mazumdar A., Michel E., Antia H. M., Deheuvels S. 2012, A\&A, 540, A31

Mazumdar A., Monteiro M. J. P. F. G., Ballot J. et al. 2014, ApJ, 782, 18

Sousa S. G., Santos N. C., Israelian G., Mayor, M. et al. 2011, A\&A, 533, A141

Verma K., Antia H. M., Basu S., Mazumdar A. 2014, ApJ 794, 114

Verma K., Raodeo K., Antia H. M., Mazumdar A. et al. 2017, ApJ, 837, 47

Verma K., Raodeo K., Basu S., Silva Aguirre V. et al., 2019, MNRAS, 483, 4678

Vorontsov S. V. 1988, IAUS, 123, 151 